\title{
(i)
}

\section{Classificação por matérias das questões do Enade e POSCOMP}

\author{
Mateus Edival Rodrigues da Silveira, Daniel Carlos Chaves Boll, Felipi Lima Matozinho, \\ Igor França Negrizoli, Leonardo Vanzin, Mateus Karvat Camara, \\ Matheus Nunes Ismael, Roberta Aparecida da Silva Alcantara, Marcio Seiji Oyamada
}

\author{
${ }^{1}$ Centro de Ciências Exatas e Tecnológicas \\ Universidade Estadual do Oeste do Paraná (UNIOESTE)
}

email: mateus.silveira, daniel.boll, felipi.matozinho, igor.negrizoli, leonardo.vanzin, mateus.camara, matheus.ismael, roberta.alcantara, marcio.oyamada @ unioeste.br

\begin{abstract}
The assessment process of undergraduate courses adopted by the Ministry of Education is the National Student Performance Exam (Enade). The National Graduate Exam for Computer Science (POSCOMP), organized by the Brazilian Computer Society, supports the admission process of students in Master's and Doctor's Degree courses. This project seeks to analyze the questions of Enade (the years of 2008, 2011, 2014, and 2017), and POSCOMP (the years of 2014, 2015, 2016, 2017, and 2018), and to classify the scope and determine which subjects are found in the questions. This project was developed aiming to achieve one of the objectives proposed in the Tutorial Education Program (PET), which is to contribute to the improvement of undergraduate courses and students. It is worth mentioning that the project does not intend to adapt the authors' Computer Science BSc to the tests, but to provide information to the course coordinator for future improvements.
\end{abstract}

Resumo. O processo de avaliação de cursos de graduação adotado pelo Ministério da Educação é o Exame Nacional de Desempenho dos Estudantes (Enade). O Exame Nacional para Ingresso na Pós-Graduação em Computação (POSCOMP), organizado pela Sociedade Brasileira de Computação (SBC), tem como objetivo possibilitar a participação em processos seletivos de cursos de mestrado e doutorado, sem a necessidade de deslocamento. Este projeto tem como objetivo analisar as questões das provas do Enade (anos de 2008, 2011, 2014 e 2017) e POSCOMP (anos de 2014, 2015, 2016, 2017 e 2018), visando determinar quais matérias são exigidas nas questões. Este projeto foi desenvolvido visando atingir um dos objetivos do Programa de Educação Tutorial, contribuindo para a elevação da qualidade da formação acadêmica dos alunos de graduação. É importante ressaltar que o trabalho proposto não tem como objetivo tentar moldar o Bacharelado em Ciência da Computação no qual o grupo PET atua ou mesmo as disciplinas para preparar os alunos para esses exames, mas tem caráter informativo e serve como ferramenta de auxílio para a coordenação do curso.

\section{Introdução}

Em 1996, foi implantado o Exame Nacional de Cursos (ENC), contemplando a exigência da Lei de Diretrizes e Bases da Educação. Na época chamado de Provão, o exame foi 
aplicado pela primeira vez para mais de 55 mil concluintes dos cursos de Administração, Engenharia Civil e Direito [INEP 2020].

O Sistema Nacional de Avaliação da Educação Superior (SINAES), estabelecido na Lei 1086/04 de 14 de Abril de 2004, substituiu o Provão, sendo composto de três eixos [Brito 2008]:

- Avaliação Institucional: auto-avaliação pela Comissão Permanente de Avaliação (CPA) e avaliação externa in loco, desenvolvida pelos avaliadores institucionais capacitados pelo INEP nos moldes do SINAES.

- Avaliação do Desempenho dos estudantes ingressantes e concluintes: através de um exame em larga escala aplicado aos estudantes que preenchem os critérios estabelecidos pela legislação vigente. O Exame Nacional de Desempenho dos Estudantes (Enade) é composto pela prova, o questionário de Avaliação Discente da Educação Superior (ADES) (antigo questionário sócio-econômico), o questionário dos coordenadores de curso e a percepção do aluno sobre a prova.

- Avaliação de Curso: pelos pares na avaliação in loco, pelos estudantes utilizando o questionário de Avaliação Discente da Educação Superior que é enviado aos estudantes da amostra do Enade, pelos coordenadores de curso, mediante questionário dos coordenadores e avaliações realizadas pelos professores dos cursos e a CPA.

O objetivo do Enade é avaliar o rendimento dos concluintes da graduação. O exame é obrigatório para os estudantes habilitados para a prova, devendo esta informação constar no histórico escolar. A periodicidade máxima da avaliação é trienal para cada área do conhecimento [INEP 2020].

Por sua vez, o Exame Nacional para Ingresso na Pós-Graduação em Computação (POSCOMP), é um exame organizado pela Sociedade Brasileira de Computação (SBC), sendo aplicado em diversas sedes espalhadas pelo país e também em alguns locais no exterior. O objetivo do POSCOMP é avaliar o conhecimento de candidatos a Programas de Pós-Graduação em Computação oferecidos no Brasil [SBC 2020].

Alguns trabalhos realizados utilizam os dados do Enade para analisar o desempenho de instituições, como ferramenta de apoio à melhoria dos cursos de graduação. O trabalho apresentado por [Figueiro et al. 2018], utiliza a técnica de clusterização dos microdados do Enade para extrair informações de qualidade de cursos de Ciência da Computação de diferentes instituições privadas do Rio Grande do Sul e criar agrupamentos de instituições de desempenho similar. Em [Francelino et al. 2020], os autores utilizam os dados do Enade 2013 dos cursos de Computação, e realizam a clusterização utilizando o método k-means. A análise relaciona o questionário socio-econômico e de notas para criar clusters. O estudo indicou que não existe uma relação entre desempenho e renda familiar, bem como desempenho e localização geográfica, sendo que as maiores notas se espalham de forma homogênea em diferentes estados do país.

Neste trabalho, diferentemente dos trabalhos citados anteriormente, a análise foi realizada em cada questão, visando classificá-las em matérias. O termo matéria foi utilizado para evitar o mapeamento em uma disciplina específica, e sim na grande área à qual o tema pertence. O objetivo foi gerar um relatório para auxiliar a coordenação do Bacharelado de Ciência da Computação no qual o grupo PET atua a ter uma visão abrangente dos temas abordados nessas avaliações, e também como um mecanismo auxiliar na melhoria da qualidade do curso. Adicionalmente, o trabalho teve como objetivo propiciar 
aos petianos o contato com provas do Enade e POSCOMP, para que eles possam sentir as diferentes faces de cada avaliação, além de proporcionar a discussão das questões em grupo. Este projeto foi desenvolvido do ano de 2017 até o primeiro trimestre de 2019, e analisou as provas do Enade (anos de 2008, 2011,2014 e 2017) e POSCOMP (anos de 2014, 2015, 2016, 2017 e 2018).

\section{Metodologia}

O projeto foi desenvolvido no grupo PETComp - Curso Ciência da Computação do Campus de Cascavel da Universidade Estadual do Oeste do Paraná. O grupo inicialmente se dividiu em três equipes, compostas por membros de séries distintas. Cada grupo analisou as provas e, para cada questão, indicou se o assunto é estudado no curso e em qual matéria. Depois da classificação inicial para cada prova, o grupo se reuniu e revisou a classificação em grupo. Nas questões discordantes, uma análise aprofundada foi realizada de forma coletiva até se atingir o consenso.

Uma das opções adotadas neste trabalho foi a escolha do termo matéria para classificação dos assuntos abordados na questão, visto que essa classificação é a mais visível e clara que os alunos têm a respeito da organização dos conteúdos e assuntos trabalhados no curso. Assim, assuntos que são abordados em sequência como Organização e Arquitetura de Computadores I e II, Processo de Engenharia de Software I e II, foram classificados nas matérias Organização e Arquitetura de Computadores e Processo de Engenharia de Software, respectivamente.

Ao final, um relatório foi gerado e apresentado aos professores e discentes membros do Colegiado, bem como ao Coordenador do Curso. O relatório também incluiu os dados do relatório de desempenho do Enade, que apresenta o desempenho dos alunos do curso de Ciência da Computação no qual este trabalho foi desenvolvido e compara com os resultados obtidos por cursos da mesma área na Unidade Federativa (UF), Região, Categoria Administrativa, Organização Acadêmica e no Brasil. Todos os relatórios, assim como as provas, estão disponíveis no repositório do grupo ${ }^{1}$.

\section{Resultados}

Nesta seção são apresentados os resultados da classificação realizada pelo grupo para o Enade e POSCOMP, respectivamente. A Tabela 1 apresenta uma lista de siglas utilizadas na apresentação de resultados.

\subsection{Enade}

A Figura 1 apresenta a distribuição das 27 questões do componente específico por disciplinas/matérias do Enade 2017. Durante a discussão, o grupo optou por classificar determinadas questões como multidisciplinar, pois sua resolução envolve conceitos de mais de uma matéria ou disciplina. Assim, duas questões foram classificadas como multidisciplinares. A multidisciplinaridade em alguns casos acontece pela própria natureza do assunto como no caso da questão que envolve conhecimentos da área de Teoria da Computação e Compiladores (TC/Comp). A questão 11 é a única que contém um conceito mais amplo de multidisciplinaridade, exigindo conceitos de Estrutura de dados, Linguagens de

\footnotetext{
${ }^{1}$ https://github.com/petcomp-unioeste/EnadePOSCOMP
} 


\begin{tabular}{|c|l||c|l|}
\hline ALG & Algoritmos & CDI & Cálculo Diferencial e Integral \\
LMD & Lógica e Matemática Discreta & LM & Linguagens de Montagem \\
PE & Probabilidade e Estatística & IS & Informática e Sociedade \\
OAC & Organização e Arquitetura de Com- & GAAL & Geometria Analítica e Álgebra Li- \\
& putadores & & near \\
ED & Estrutura de Dados & SD & Sistemas Digitais \\
PES & Processo de Engenharia de Software & DIST & Sistemas Distribuídos \\
CG & Computação Gráfica & PID & Processamento de Imagens Digital \\
SO & Sistemas Operacionais & BD & Banco de Dados \\
IA & Inteligência Artificial & LP & Linguagens de Programação \\
TC & Teoria da Computação & PAA & Projeto e Análise de Algoritmos \\
RC & Redes de Computadores & Comp & Compiladores \\
ICC & Introdução à Ciência da Computação & & \\
\hline
\end{tabular}

Tabela 1. Sigla das Matérias

Programação e Engenharia de Software (ED/LP/PES). É possível notar que existe uma divisão equilibrada de matérias abordadas, com 1 ou 2 questões. A única exceção nesta edição foi a matéria de Sistemas Operacionais com 3 questões.

\section{ENADE 2017}

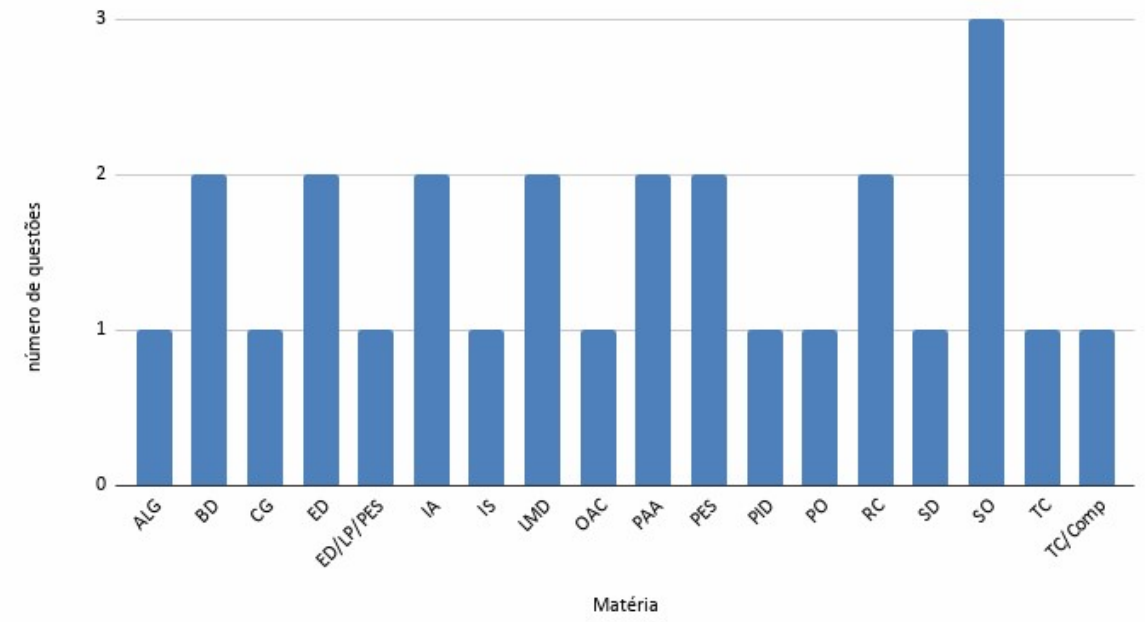

Figura 1. Distribuição das questões em matérias no Enade 2017

A Figura 2 apresenta a distribuição das 27 questões do componente específico por disciplinas/matérias do Enade 2014. Das 27 questões analisadas, 4 questões foram classificadas como multidisciplinares, sendo que em alguns casos existe uma relação forte como o caso de Estrutura de Dados e Projeto e Análise de Algoritmos (ED/PAA). A questão 22 é a única que contém um conceito mais amplo de multidisciplinaridade, exigindo conceitos de Estrutura de Dados, Pesquisa Operacional, Inteligência Artificial e Projeto e Análise de Algoritmos (ED/PO/IA/PAA). Nota-se que Inteligência Artificial e Sistemas Operacionais possuem a maior concentração de questões, com 3 e 4 respectivamente.

A Figura 3 apresenta a distribuição das questões do Enade 2011 por disciplinas/matérias no total de 27 questões. Podemos notar que o número de questões que foram 


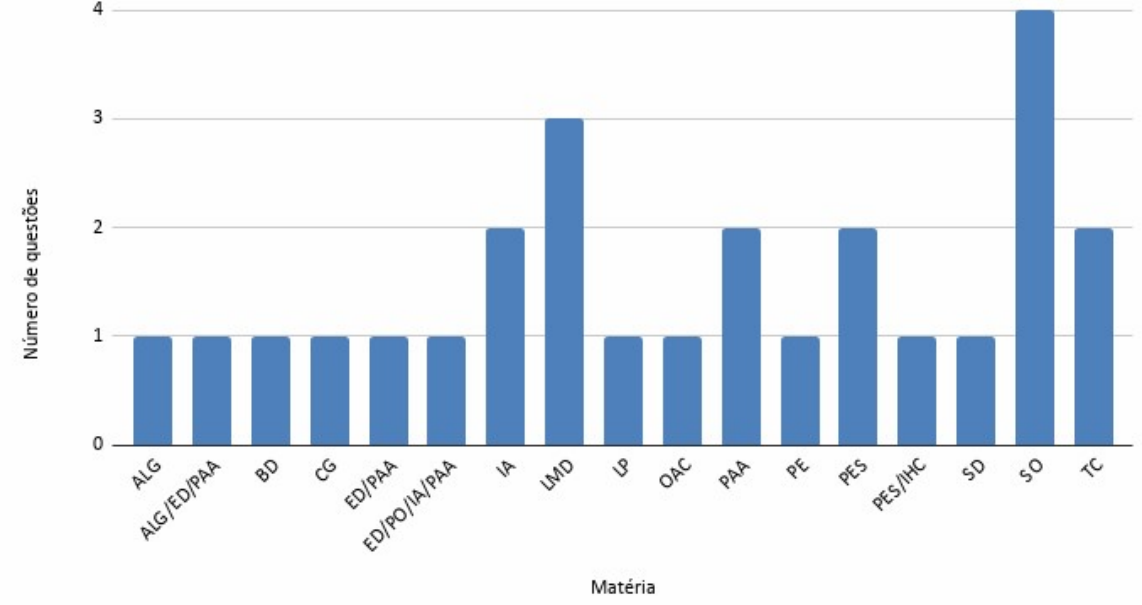

Figura 2. Distribuição das questões em matérias no Enade 2014

classificadas como multidisciplinares é baixo. Adicionalmente, a multidisciplinaridade envolve assuntos correlatos, possibilitando que o aluno possa naturalmente relacionar as matérias e resolver a questão. Uma matéria adicionada nesta etapa foi a Distribuídos e Paralelos (DIST_PAR), que, em alguns anos no curso onde o grupo PET atua, é oferecida como optativa. Nesta edição, verifica-se a matéria Teoria da Computação com a maior concentração de questões, totalizando 4 questões.

\section{ENADE 2011}

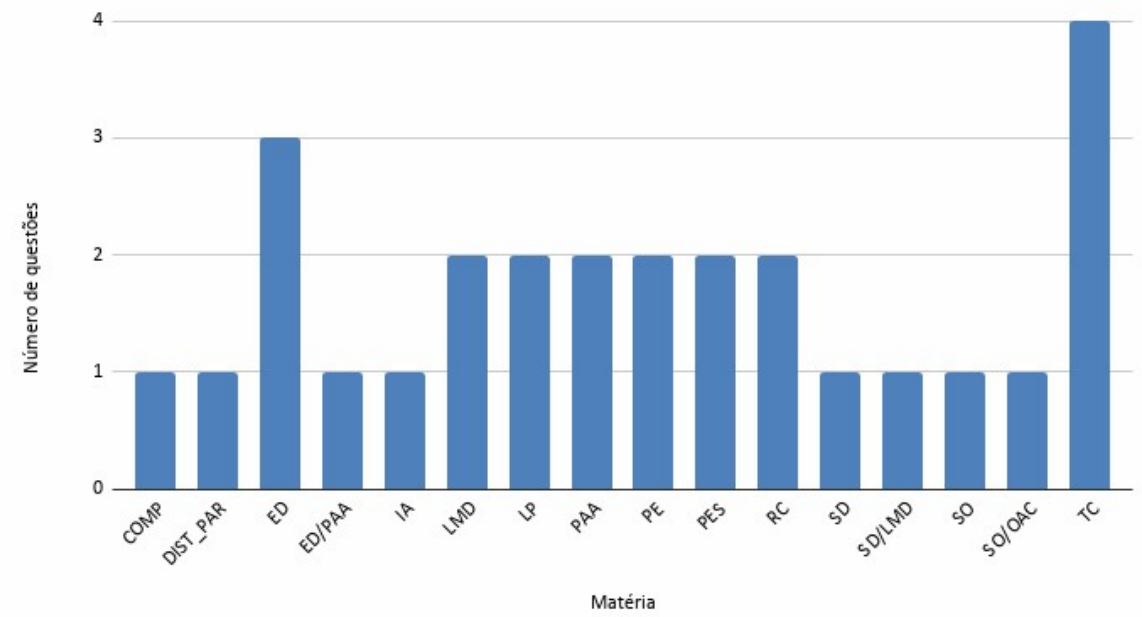

Figura 3. Distribuição das questões em matérias no Enade 2011

A Figura 4 apresenta os resultados da divisão de matérias no Enade 2008, em um total de 31 questões do componente específico. Existe um balanceamento na distribuição de questões, exceto pela matéria de Redes de Computadores, com 5 questões na prova. 


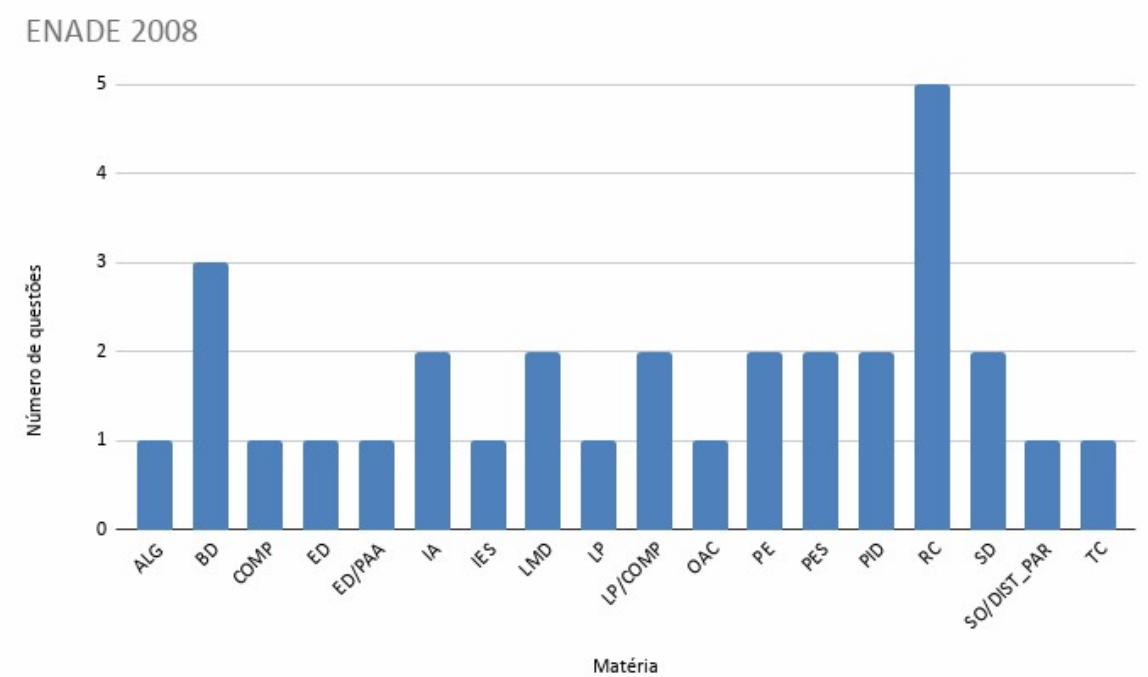

Figura 4. Distribuição das questões em matérias no Enade 2008

\subsection{POSCOMP}

A Figura 5 apresenta os dados obtidos do POSCOMP 2018. O POSCOMP contém 70 questões, sendo 20 de Matemática, 30 de Fundamentos da Computação e 20 de Tecnologia da Computação. $\mathrm{Na}$ análise da prova nota-se a característica mais compartimentalizada e definida em matérias. Apenas uma questão se caracteriza de forma multidisciplinar, envolvendo conceitos de Linguagens de Programação e Processo de Engenharia de Software (LP/PES). Na distribuição de questões, destaca-se Estrutura de Dados com 8 questões. Essa característica era esperada pois a prova tem como objetivo avaliar candidatos para programas de pós-graduação em Ciência da Computação e visa avaliar a base em Fundamentos da Computação.

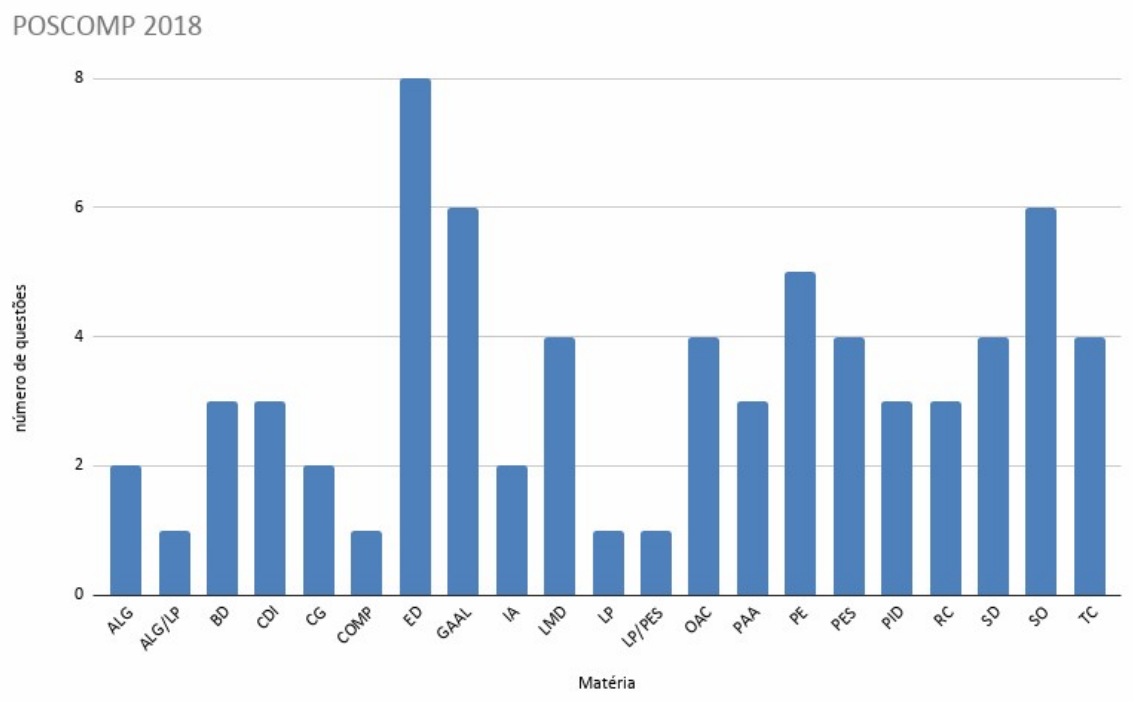

Figura 5. Distribuição das questões em matérias no POSCOMP 2018

A Figura 6 apresenta o resultado da classificação do POSCOMP 2017. A prova 
tem um total de 70 questões, com a mesma distribuição do POSCOMP 2018. Nesta prova, novamente algumas questões foram classificadas como a junção de diferentes matérias, tais como Redes de Computadores e Sistemas Distribuídos (RC/DIST), e Algoritmos e Linguagens de Programação (ALG/LP). Nota-se novamente a característica da prova com questões concentradas na matéria Estrutura de Dados.

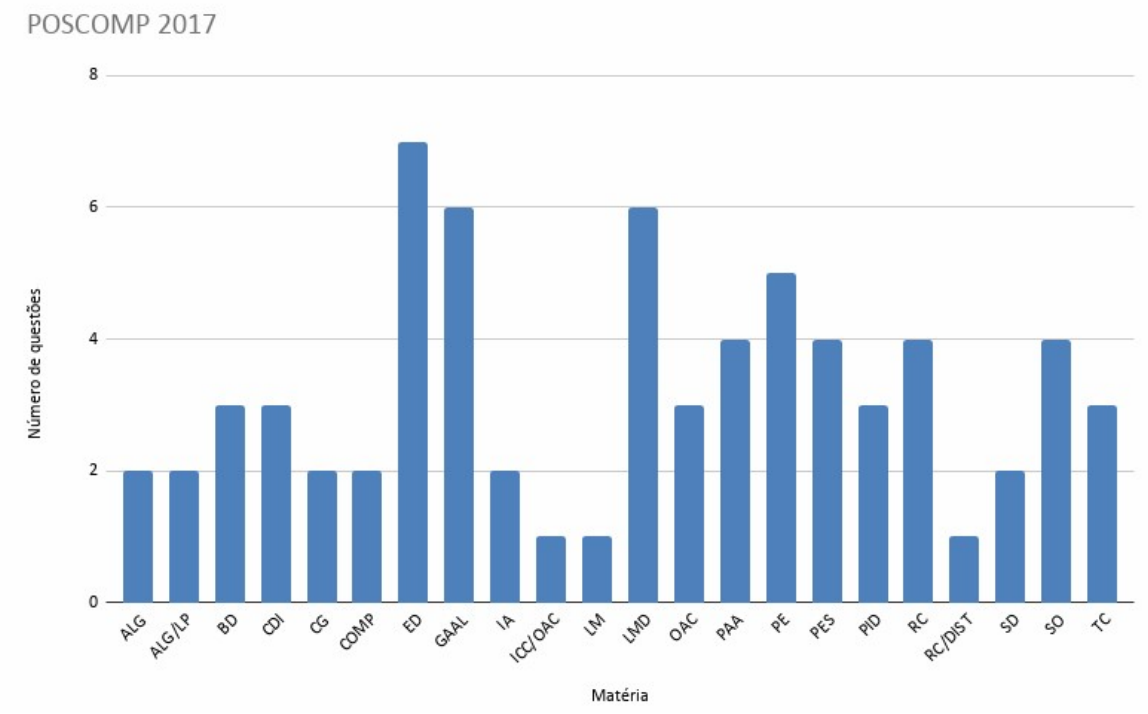

Figura 6. Distribuição das questões em matérias no POSCOMP 2017

A Figura 7 apresenta o resultado da classificação do POSCOMP 2016. No processo de classificação dessa prova, o grupo decidiu criar 4 novas matérias, pois avaliou que assim ficaria mais claro definir de qual assunto a questão abordava. As matérias criadas foram: Teoria dos Grafos (TG), Tolerância a Falhas (TF), Banco de Dados Distribuídos (BDD) e Java. A matéria Java foi criada pois notou-se que a questão exigia conceitos específicos da linguagem para sua resolução. Outra característica desta edição foi o balanceamento das questões entre as diferentes matérias da computação. A concentração ficou mais evidente para as matérias de Matemática, como Geometria Analítica e Álgebra Linear (GAAL), Lógica e Matemática Discreta (LMD), e Probabilidade e Estatística (PE).

A Figura 8 apresenta os dados obtidos do POSCOMP 2015. Nele, o grupo classificou uma questão como $A D-H O C$, pois houve dificuldade em classificá-la em alguma disciplina ou matéria. A questão avaliava se o armazenamento de informações em metadados no arquivo facilitava a portabilidade. Um outro ponto a ser notado é que uma questão foi classificada como necessitando de conhecimento de Física, pois envolvia conceitos de Mecânica. No entanto, é importante frisar que tal conteúdo normalmente não é coberto nas disciplinas de Física de cursos de Ciência da Computação no Brasil. Foi criada também a matéria denominada de Programação Orientada a Objetos (POO), por considerar-se que este assunto é transversal, sendo abordado em mais de uma disciplina nos cursos de Ciência da Computação.

A Figura 9 apresenta os dados obtidos do POSCOMP 2014. Nessa prova, o grupo classificou uma questão como LISP, pois a questão abordava conceitos muitos específicos da linguagem. Nota-se também a concentração de questões na matéria Estrutura de Dados. 


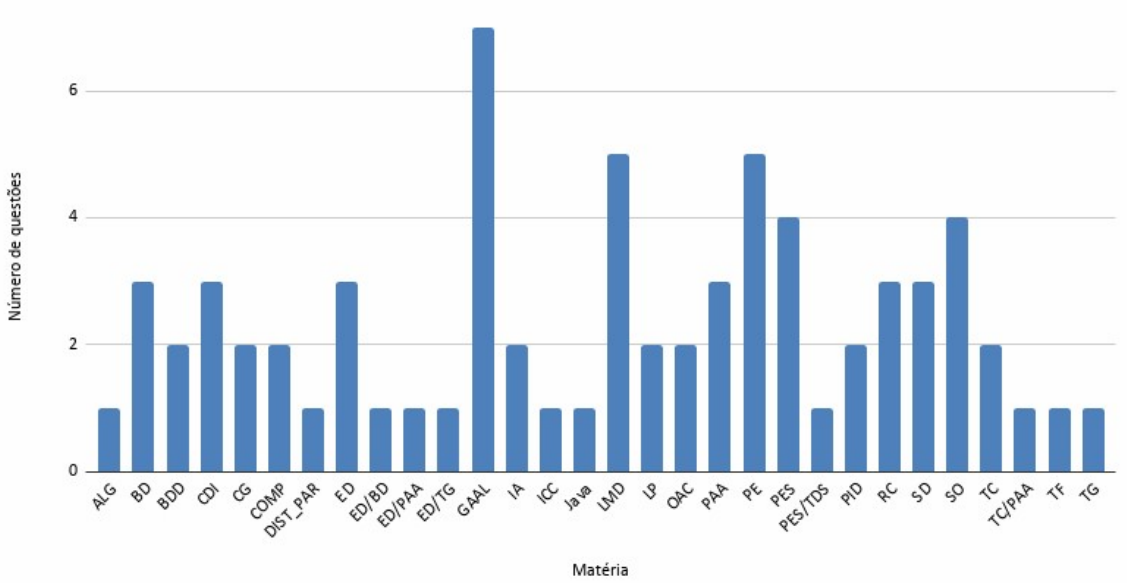

Figura 7. Distribuição das questões em matérias no POSCOMP 2016

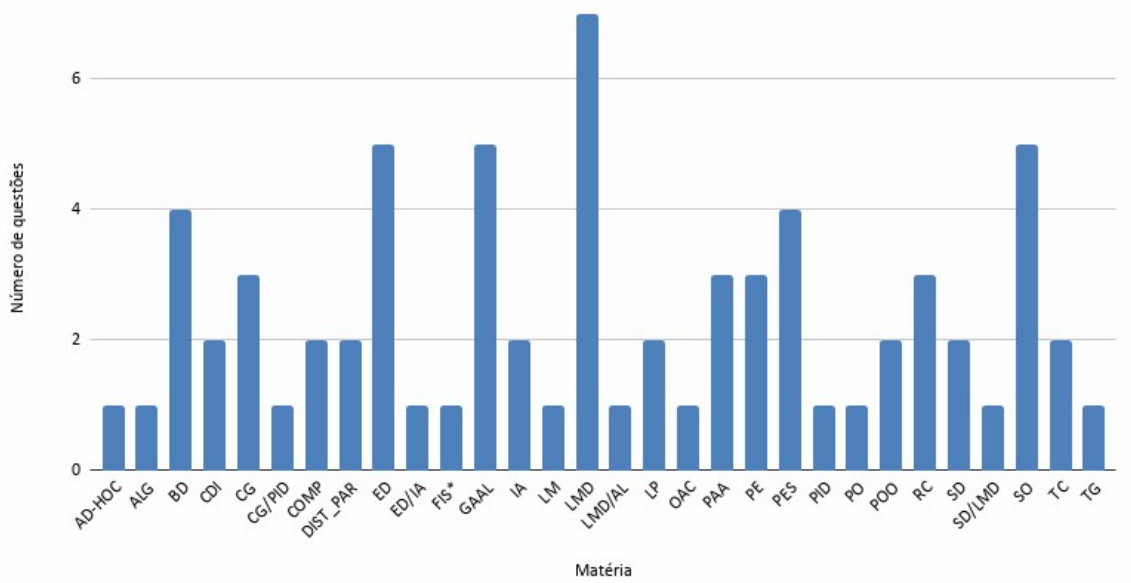

Figura 8. Distribuição das questões em matérias no POSCOMP 2015

Com base na análise de cada uma destas provas, organizou-se a Tabela 2, a qual apresenta o número de diferentes matérias que foram coletadas em cada prova. Pode-se notar que ao longo das edições do Enade, o número de diferentes matérias se manteve muito próximo. No caso do POSCOMP, é possível notar que até 2016 a prova era mais abrangente, sendo que durante o processo o grupo criou matérias como Teoria dos Grafos, Tolerância a Falhas, Pesquisa Operacional, Banco de Dados Distribuídos, Java e Lisp. No entanto, a partir de 2017 verificou-se uma redução no número de matérias distintas. É importante ressaltar que no POSCOMP foram classificadas também as questões da área de Matemática, portanto o número maior de matérias em comparação com o Enade era esperado. 


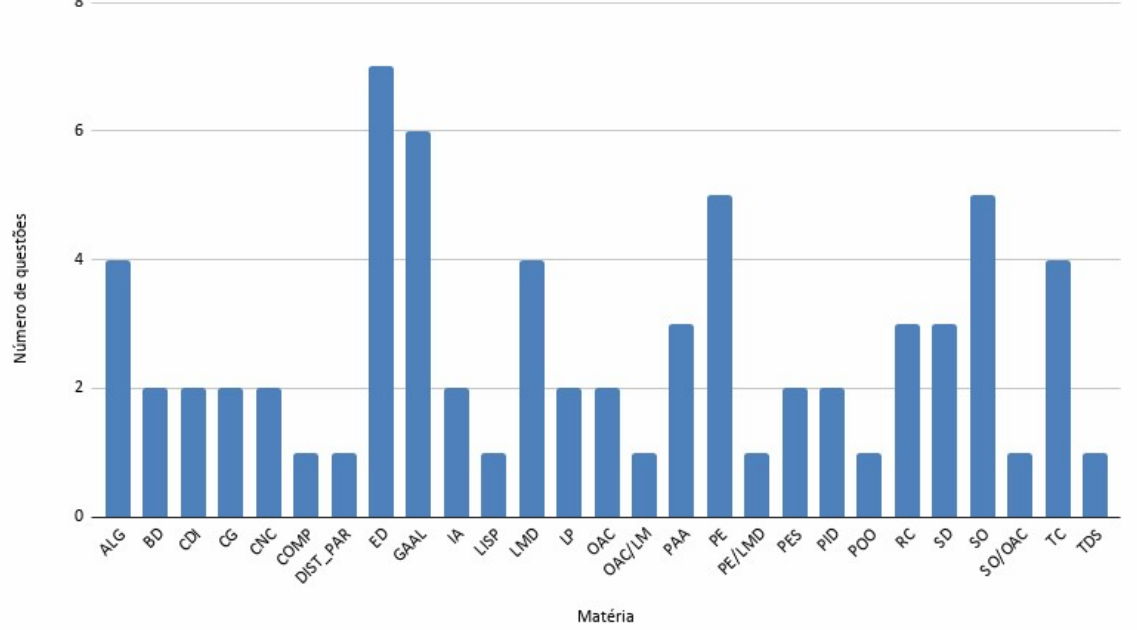

Figura 9. Distribuição das questões em matérias no POSCOMP 2014

\begin{tabular}{|l|c|} 
Número de questões & Número de matérias distintas \\
\hline Enade 2008 & 18 \\
Enade 2011 & 16 \\
Enade 2014 & 16 \\
Enade 2017 & 17 \\
POSCOMP 2014 & 26 \\
POSCOMP 2015 & 29 \\
POSCOMP 2016 & 29 \\
POSCOMP 2017 & 21 \\
POSCOMP 2018 & 20
\end{tabular}

Tabela 2. Número de matérias distintas por prova

\section{Conclusões e Trabalhos futuros}

Inicialmente, são apresentadas algumas opiniões dos alunos que participaram do projeto.

"Nota-se que em ambos os exames a interpretação desenvolve um importante papel ao identificar a correta alternativa para uma determinada questão. Claramente, durante as revisões das provas realizadas pelas equipes, notou-se que ocorriam diferentes entendimentos sobre a abordagem de uma determinada pergunta. Dessa forma, reforça-se que o estímulo recebido pelo aluno durante o curso, forçando-o a visualizar um determinado conceito em uma situação inusual, é crucial para que seja fornecida uma boa formação."

"Com o conhecimento adquirido de maneira geral durante o curso é possível responder questões sobre assuntos que não foram abordados, através de interpretação e conhecimento base, sobretudo nas provas do Enade. A maior dificuldade, percebida nas provas do POSCOMP, foi em lembrar conceitos vistos, por exemplo, nas aulas de Geometria Analítica e Álgebra Linear, que são retomados na matéria de Computação Gráfica." 
"Em relação ao estilo de prova, percebe-se que o POSCOMP é uma prova mais técnica, com diversas questões exigindo conhecimentos pontuais de uma disciplina específica. Já o Enade tenta realizar uma avaliação mais abrangente, o que é observado tanto no estilo das questões, que buscam relacionar conceitos diversos, como no próprio estilo da prova, incluindo questões abertas além de questões de formação geral."

"Como aluno do primeiro ano, pude ter um panorama amplo de todas as matérias estudadas no curso, podendo notar como elas se relacionam entre si, mas também como dependem de matérias estudadas já no primeiro ano, como Lógica e Matemática Discreta e Algoritmos."

"Como estudante do terceiro ano, iniciando os estudos para esse tipo de exame, o projeto auxiliou aos que não conheciam as provas saber mais sobre, como elas funcionam, qual a importância delas no âmbito acadêmico."

"Esse projeto me proporcionou a oportunidade de entrar em contato com as disciplinas já concluídas, assim, podendo reafirmar meus conhecimentos sobre tais disciplinas."

Assim, baseado nos resultados e observações levantadas, após a construção do relatório o grupo pôde concluir:

- Podemos notar que não existe uma distribuição com alguma disciplina/matéria predominante. Assim, é inviável moldar o projeto político pedagógico baseado exclusivamente em algumas das avaliações.

- O núcleo de disciplinas considerado base na Computação é sempre encontrado em todas as provas, confirmando a importância de tais componentes na formação sólida em Ciência da Computação.

- As provas são abrangentes, portanto a formação sólida e exigente nas disciplinas e trabalhos é a melhor forma de obter um bom desempenho nessas avaliações. A preparação somente para esses exames com cursinhos ou revisões pode não ser uma estratégia adequada.

- A matéria de Física é inexistente nas provas analisadas exceto por uma única questão de Mecânica, que não é abordada em cursos de Computação.

- A multidisciplinaridade é pouco encontrada no POSCOMP. No Enade, tal conceito é mais presente, exigindo habilidade na interpretação de texto e a aplicação de conceitos computacionais em contextos distintos dos vistos nas disciplinas.

- Possuir uma boa base matemática é essencial para uma boa interpretação das questões apresentadas nas provas.

O relatório gerado foi apresentado para o Colegiado do curso durante o final do processo de criação do último Projeto Político Pedagógico. Os resultados reafirmaram a necessidade de uma carga horária e formação abrangente nas disciplinas de Fundamentos da Computação, proporcionando uma base sólida aos estudantes. Além disso, baseado nas Diretrizes curriculares de Cursos de Computação [MEC 2020], o Colegiado havia decidido pela retirada de Física da grade do curso. O relatório deste projeto foi apresentado após essa decisão e corroborou a alternativa adotada pelo Colegiado do curso.

No futuro pretende-se continuar com a análise das provas futuras do Enade e POSCOMP para geração de novos relatórios para comparação com os dados obtidos anteriormente. Pretende-se também abrir o projeto para os demais alunos do curso, proporcio- 
nando que alunos que se interessem por essa avaliação tenham contato com a prova e suas características.

\section{Agradecimentos}

Ao MEC/SESU pelas bolsas do grupo PETComp. Aos petianos egressos: Alexandre Barreiro Neto, Camila Tiemi Outa, Danielly Omori Antunes de Oliveira, Lucas Frank Hollmann, Elixandre Michael Baldi, Gabriel Bruscatto, Guilherme Zabot, Hendric Gabriel Cechinato, Henrique Oliveira, Letícia Torres Bertoldo, Luis Fernando Veronese Trivelatto, Matheus de Lara Dias da Silva, Matheus Leonardo da Silva Dias, Nicolas Afonso Bertaglia Comissio, Valquíria Prestes Belusso, Vinícius Laurenci, Willian Cavaller Faino pela participação na classificação das questões.

\section{Referências}

Brito, M. R. (2008). O SINAES e o ENADE: da concepção à implantação. Avaliação: Revista da Avaliação da Educação Superior (Campinas), 13:841 - 850.

Figueiro, M., Vista, N., Barasuol, J., Chicon, P., and Ansuj, A. (2018). Análise de agrupamento hierárquico aplicada aos microdados do enade do curso de graduação em ciência da computação. Revista Eletrônica Argentina-Brasil de Tecnologias da Informação e da Comunicação, 1(8).

Francelino, W. L., Savio, L. J., and de Souza Machado, L. (2020). Mineração de dados nos microdados ENADE Computação. https://riuni.unisul.br/handle/12345/9988. 17 de outubro, 2020.

INEP (2020). ENADE- exame nacional de desempenho dos estudantes. $\quad$ https://www.gov.br/inep/pt-br/areas-de-atuacao/avaliacao-e-exameseducacionais/enade. 17 de outubro, 2020.

MEC (2020). Diretrizes Curriculares Nacionais para os cursos de graduação na área da Computação. http://portal.mec.gov.br/component/content/article?id=12991. $17 \mathrm{de}$ outubro, 2020.

SBC (2020). POSCOMP- Exame Nacional para Ingresso na Pós-Graduação em Computação. https://www.sbc.org.br/poscomp. 17 de outubro, 2020. 